myeloid-derived suppressor cell (MDSC) is pivotal for immunosuppression, we investigated its role and regulation in the fibrotic microenvironment with an aim of developing mechanism-based combination immunotherapy.

Methods Functional significance of MDSCs was evaluated by flow cytometry using two orthotopic HCC models in fibrotic liver setting via carbon tetrachloride or high-fat high-carbohydrate diet, and verified by clinical specimens. Mechanistic studies were conducted in the human hepatic stellate cell (HSC)-peripheral blood mononuclear cell culture systems and fibrotic-HCC patient-derived MDSCs. The efficacy of single or combined therapy with anti-programmed death-1-ligand-1 (anti-PD-L1) and a clinically-trialed BET bromodomain inhibitor i-BET762 was determined.

Results Accumulation of monocytic MDSCs (M-MDSCs), but not polymorphonuclear MDSCs, in fibrotic livers significantly correlated with reduced tumor-infiltrating lymphocytes (TILs) and increased tumorigenicity in both mouse models. In human HCCs, the tumor-surrounding fibrotic livers were markedly enriched with M-MDSC, with its surrogate marker CD33 significantly associated with aggressive tumor phenotypes and poor survival rates. Mechanistically, activated HSCs induced monocyte-intrinsic p38 MAPK signaling to trigger enhancer reprogramming for M-MDSC development and immunosuppression. Treatment with p38 MAPK inhibitor abrogated HSCM-MDSC crosstalk to prevent HCC growth. Concomitant with patient-derived M-MDSC suppression by i-BET762, combined treatment with anti-PD-L1 synergistically enhanced TILs, resulting in tumor eradication and prolonged survival in the fibrotic-HCC mouse model.

Conclusions Our results signify how non-tumor intrinsic properties in the desmoplastic microenvironment can be exploited to reinstate immunosurveillance, providing readily translatable combination strategies to empower HCC immunotherapy (figure 1).

Acknowledgement This project is supported by the University Grants Committee through the Collaborative Research Fund (C4045-18W) and the Theme-based Research Scheme (T11706/18-N), the Health and Medical Research Fund (16170451), the Terry Fox Foundation, the Focused Innovations Scheme-Scheme B (1907309) from the Chinese University of Hong Kong and the Li Ka Shing Foundation (Canada). Man Liu is supported by China Postdoctoral Science Foundation.

\section{IDDF2019-ABS-0299 GUT MICROBIOME DIVERSITY AND SPECIFIC MICROBIAL GENERA CORRELATE WITH THE SEVERITY OF NON-ALCOHOLIC LIVER DISEASE IN INDONESIA}

\begin{abstract}
${ }^{1}$ Chyntia Olivia Maurine Jasirwan*, ${ }^{1}$ Cosmas Rinaldi Adhitya Lesmana, ${ }^{1}$ Irsan Hasan, ${ }^{2}$ Marcellus Simadibrata, ${ }^{3} \mathrm{k}$ hwan Rinaldi, ${ }^{1} J u f e r d y$ Kurniawan, ${ }^{1}$ Andri Sanityoso, ${ }^{1}$ Saut Nababan, ${ }^{1}$ Rino Alvani Gani. 'Hepatobiliary Division, Department of Internal Medicine, Dr. Cipto Mangunkusumo Hospital, Universitas Indonesia, Jakarta, Indonesia; ${ }^{2}$ Gastroenterology Division, Department of Internal Medicine, Dr. Cipto Mangunkusumo Hospital, Universitas Indonesia, Jakarta, Indonesia; ${ }^{3}$ Clinical Epidemiology Unit and Hematology and Oncology Division, Department of Internal Medicine, Dr. Cipto Mangunkusumo Hospital, Universitas Indonesia, Jakarta, Indonesia
\end{abstract}

\subsection{6/gutjnl-2019-IDDFabstracts.5}

Background The prevalence of nonalcoholic fatty liver disease (NAFLD) is increasing. In Indonesia Hasan, I et al. (2002) reported the prevalence of $30.6 \%$. This study evaluated the first time the profile of gut microbiota and correlation with severity of NAFLD in Indonesia.

Methods We included 37 subjects age 18-60 years. The characteristic data of the patients and the food recalls were recorded. Abdominal ultrasound, liver transient elastography with controlled attenuation parameter (CAP) were performed. Next generation 16S rRNA metagenomic sequencing was conducted using stool samples. The Spearman correlation test was used to examine the correlation between specific microbial taxa with the severity of NAFLD based on fibrosis and steatosis degree.

Results The subjects included 62.2\% $(n=23)$ females and $37.8 \%$ males $(n=14)$, mean age $50 \pm 7.93$ years old. They were divided based on fibrosis and steatosis degree into nonsignificant and significant fibrosis using cut off $7 \mathrm{KPa}$, mild and moderate-severe steatosis based on cut off $270 \mathrm{~dB} / \mathrm{m}$. From all subjects, we got $73 \%$ vs $27 \%$ non-significant and significant fibrosis, $51.4 \%$ vs $48.6 \%$ mild and moderatesevere steatosis. At the phylum level, the proportion of Bacteriodetes did not change in fibrosis or steatosis group. The proportion of Proteobacteria and Firmicutes was different in fibrosis and steatosis groups. Actinobacteria unknown bifidobacteriales bifidobacteriaceae bifidobacterium Bifidobacterium adolescentis correlate positively with non significant fibrosis $(\mathrm{r}=0.532 ; \mathrm{p}=0.004)$. Firmicutes clostridia clostridiales lachnospiraceae unknown fusicatenibacter saccharivorans and Firmicutes clostridia clostridales ruminococcaceae unknown [clostridium] leptum correlate negatively with significant fibrosis $(\mathrm{r}=-0.695 ; \mathrm{p}=0.026$ vs $\mathrm{r}=-0.732 ; \mathrm{p}=0.016)$. In the group of steatosis, we got Bacteroidetes bacteroidia bacteroidales rikenellaceae alistipes alistipes onderdonkii and Firmicutes clostridia clostridales oscillospiracea oscillospiraceao scilibacter ruminantum correlate negatively with moderatesevere steatosis $(\mathrm{r}=-0.478 ; \mathrm{p}=0.045$ vs $\mathrm{r}=-0.518$; $\mathrm{p}=0.028)$; Bacteroidetes bacteroidia bacteroidales rikenellaceae alistipes alistipes putredinis correlate positively with mild steatosis ( $\mathrm{r}=0.503 ; \mathrm{p}=0.028)$.

Conclusions The abundance of microbiota in NAFLD are not significantly different based on the group of fibrosis and steatosis, especially in phylum level. But at the lower level, some specific microbiota may correlate with the degree of fibrosis and steatosis.

\section{Clinical Gastroenterology}

\section{IDDF2019-ABS-0042 PROSPECTIVE STUDY OF RISK SCORE STRATEGIES IN THE PREDICTION OF ADVANCED COLORECTAL NEOPLASIA AT COLONOSCOPY}

${ }^{1}$ Bianca So*, ${ }^{2}$ Yunki Yau, ${ }^{2}$ Viraj Kariyawasam, ${ }^{3}$ Rupert Leong. ${ }^{1}$ Faculty of Medicine, University of New South Wales, Australia; ${ }^{2}$ Gastroenterology and Liver Services, Concord Repatriation General Hospital, Australia; ${ }^{3}$ Sydney Medical School, University of Sydney, Australia

\subsection{6/gutjnl-2019-IDDFabstracts.6}

Background Current referral pathways in Australia for colorectal cancer (CRC) screening do not differentiate well between low and high-risk populations, and therefore may not be efficiently utilising resources. Whilst multiple CRC risk scoring systems currently exist and are utilised to stratify patients into low and high risk groups for priority of 